WIENER SLAVISTISCHES JAHRBUCH, Band 56/2010, 141-160

(C) 2010 by Österreichische Akademie der Wissenschaften, Wien

Alois Wold A N

\title{
Ivan Mazepa in der deutschsprachigen Literatur
}

Im Jahr 2009 jährte sich zum 300. Mal ein historisches Ereignis, das die Entwicklung in Nord-, Mittel und Osteuropa entscheidend beeinflusst hat (wenn man Voltaire, dem ersten Historiker, der darauf explizit eingegangen ist, Glauben schenken darf) - die Schlacht von Poltava, in der Peter d. Große über Karl XII. und die mit ihm verbündeten Teile der ukrainischen Kosaken unter dem Hetman Ivan Mazepa siegte, aber auch der 300. Todestag des Hetmans Ivan Mazepa ${ }^{1}$. Es ist deshalb nicht verwunderlich, dass sich in diesem Zusammenhang verschiedene nationale Gedächtnistraditionen auf dieses Ereignis bzw. dessen Protagonisten besinnen, wenngleich in unterschiedlicher Art und Weise. So arbeitet etwa schon seit 2007 eine gemischte schwedisch-amerikanisch-ukrainische archäologische Kommission im Auftrag der schwedischen Regierung daran, die Gräber der damals gefallenen Schweden aufzufinden. In der Ukraine wiederum hatte Präsident Juščenko mit einem besonderen Erlass zu Vorbereitungen zu diesem Gedenkjahr aufgerufen (Juščenko 2007), und man hat eine eigene Internet-Plattform eingerichtet, auf der eine Fülle von Materialien erschienen sind (www.mazepa.name). In Deutschland brachte lediglich die Wochenzeitung Die Zeit einen längeren Artikel über die Schlacht von Poltava, der auch auf die Spannungen zwischen Russland und der Ukraine in Bezug auf die 300Jahr Feier und vor allem auf geplante Denkmäler für die Verlierer, Hetman Mazepa und Karl XII. von Schweden, hinweist (Geyer 2009).

Mazepa war in deutschen Landen vor dreihundert Jahren kein Unbekannter, ganz im Unterschied zu heute. Er ist wohl der bekannteste Ukrainer an der Wende vom 17. zum 18. Jahrhundert überhaupt. Dazu haben wesentlich deutsche und auch österreichische (neben englischen und französischen) Zeitungen und Journale beigetragen, die über den Kosakenhetman als Türkenhelden berichteten, Jahre bevor er auf

${ }^{1}$ Die Schreibung „Mazepa“ bezieht sich auf die historische Persönlichkeit sowie auf die literarische Figur aus Puškins Poltava, während die Schreibung „Mazeppa“ sich auf diverse literarische Gestalten bezieht. 
die schwedische Seite überwechseln sollte (Mac'kiv 1988). Auch die europäische Diplomatie war an Mazepa in höchstem Maß interessiert, wie etwa die Berichte westeuropäischer Botschafter am Moskauer Zarenhof beweisen (z. B. die der österreichischen Gesandten Baron Korb und Otto von Pleyer - Sičyns'kyj 1942: 114118). Das österreichische Interesse an Mazepa wurde noch durch einen anderen Umstand gesteigert: Mazepa sollte - auf Betreiben Peters I., mit dem er damals noch beste Beziehungen unterhielt - in den Stand eines Fürsten des Hl. Römischen Reichs erhoben werden. Das Schreiben an den Kaiser Joseph I., in dem Mazepa um diesen Titel bittet, findet sich im Haus-, Hof- und Staatsarchiv in Wien. Es ist in deutscher Sprache abgefasst und enthält eine interessante Begründung: Mazepa und seine Kosaken hätten immer die Feinde der Christenheit von deren Grenzen abgehalten und wären auch in Zukunft bereit, als eine „Vormauer der gantzen Christenheit [sich] willig bey allen Vorfallenheiten gebrauchen [zu] lassen“ (Mac'kiv 1988: 241). Hier fällt das Argument des ,antemurale christianitatis“, das wir auch aus der ungarischen, kroatischen und polnischen Selbstdarstellung der Barockzeit kennen und das in Wien - gut zwanzig Jahre nach der Zweiten Türkenbelagerung - wohl noch gewirkt hat. Mazepa wurde diese Würde tatsächlich verliehen, er hat aber davon nichts mehr erfahren, denn der Bescheid wurde nicht zugestellt, auf Betreiben des russischen Zaren, der nach dem Wechsel Mazepas auf die schwedische Seite das zu verhindern wusste. Dennoch wird Mazepa von vielen Berichten aus dieser Zeit als „Fürst" tituliert.

Die „Zweite“, literarische Karriere Mazepas beginnt im frühen 19. Jahrhundert, gut hundert Jahre nach Poltava. 1819 veröffentlichte Lord Byron sein Poem Mazep$p a$ in seiner Sammlung Oriental Tales, das sehr rasch in verschiedene europäische Sprachen übersetzt wurde; 1829 antwortete darauf zum einen Victor Hugo mit seinem Mazeppa aus der Reihe Les Orientales und Aleksandr Puškin mit seiner Dichtung Poltava, die ursprünglich Mazepa heißen sollte. 1840 publiziert der polnische Romantiker Juliusz Słowacki in Paris sein Drama Mazepa. Um die Mitte des 19. Jhds. erscheinen die ersten italienischen und deutschen Mazepa-Bearbeitungen, erst gegen Ende des 19. Jhds. und auch noch im 20. kommt es zu ukrainischen literarischen Varianten des Mazepa-Stoffs.

Lang vorher hatte aber schon die Bildende Kunst diesen Stoff aufgegriffen, die französischen Maler Théodore Gericault, Eugène Delacroix, Horace Vernet und Louis Boulanger hatten schon in den 1820er Jahren Zeichnungen und Gemälde angefertigt, welche das illustrieren, was Byron im Hauptteil seines Poems beschreibt: der schöne Jüngling Mazepa, zur Strafe für sein Verhältnis mit einer polnischen Dame nackt auf den Rücken eines wilden Pferdes gebunden, erlebt einen Ritt auf Leben und Tod, bis dass das Pferd in der Ukraine zusammenbricht und Mazepa von Kosaken gerettet wird. Genau dieses Thema greift in der Musik Franz Liszt auf - in seiner Etüde Nr. 4 für Klavier, Mazeppa (1851), gestaltet er diesen Ritt in einer Programmmusik, in seinem Poème symphonique Nr. 6 Mazeppa (1854) wird dasselbe Material zu einem großen, effektiven Orchesterstück ausgebaut. 1883 schließlich 
wird Petr I. Čajkovskijs Oper Mazepa uraufgeführt, die aber nicht auf der englischfranzösischen Version des Mazepa-Stoffs, sondern auf Puškins Poltava beruht: der alte Hetman Mazepa hat die Tochter seines Jugendfreundes Kočubej, Marija, entführt und zu seiner Geliebten gemacht, der Vater rächt sich, indem er Mazepas politische Absichten nach Moskau denunziert. Čajkovskij geht bei seiner dritten Oper nach Vorlagen von Puškin auf dessen ursprüngliches Konzept zurück (vgl. den Titel Mazepa und nicht Poltava), die Schlacht von 1709, die bei Puškin den ganzen dritten Teil seines Poems einnimmt, ist auf ein instrumentales Zwischenspiel reduziert.

Die meisten dieser prominenten Mazepa-Varianten wurden in der Fachliteratur ausführlich behandelt, die deutschsprachigen Mazepa-Bearbeitungen sind zwar bibliographisch erfasst und im internationalen Kontext erwähnt, sie wurden aber bislang nie ausführlicher analysiert. Das hängt auch damit zusammen, dass deren Autoren heute so gut wie vergessen sind. Dramatiker wie Andreas May, Karl Kösting und Rudolf v. Gottschall oder Erzähler wie Wilhelm Anthony, Adolf Mützelburg und Gustav Nieritz sind heute nur noch in Spezial-Nachschlagewerken zu finden. Trotzdem scheint es lohnenswert, einige dieser Texte dem Vergessen entreißen und in den Kontext der internationalen Mazepa-Literatur zurückführen, weil sich von daher auch Anknüpfungen an die heutige Bewertung Mazepas in der ukrainischen Belletristik ergeben.

Den „deutschen Mazepa“ kennt die ukrainische Wissenschaft schon seit gut hundert Jahren. 1904 hatte Vasyl’ Levyc'kyj eine Skizze unter dem Titel „,Der Hetman Iwan Mazepa in der deutschen Literatur" (Lewickyj 1904) publiziert, 1909, im Jahr der 200. Wiederkehr der Schlacht von Poltava, verfasste der schwedische Slawist Alfred Jensen, bekannt auch aufgrund seines Buches über Taras Ševčenko, einen Aufsatz „Mazepa in der modernen europäischen Dichtung“ (Jensen 1909), der sich nicht mehr nur auf die deutschen Fassungen beschränkte. 1917, noch vor Ende des Ersten Weltkriegs, veröffentlichte der berühmte Publizist und spätere Ideologe der ukrainischen Rechten, Dmytro Doncov, einen Artikel „Het'man Mazepa v jevropejs'kij literaturi““ (Doncov 1917), der ein Jahr später in leicht veränderter deutscher Fassung (,Mazeppa in der Weltliteratur“ - Donzow 1918) erschien. Hier gibt Doncov bereits eine Interpretation Mazepas aus dem Geist der Zeit des Ersten Weltkrieges: „In den weiten, mit dem Blut der Kämpfer für die Freiheit ihres Landes getränkten Steppen der Ukraine, in denen einst das Echo der Poltawaschlacht ertönte, rollte wieder dumpf der Kanonendonner. Geht der Geist Mazeppas wieder um?“ (Doncov 1918: 27).

Eine weitere Arbeit zu Mazepa in der Weltliteratur und Kunst („Het'man Ivan Mazepa v svitovij literaturi ta mystectvi“ - Muchyn 1932) von Mychajlo Muchyn stammt aus den Kreisen der ukrainischen Emigration in Prag - der Verfasser scheint seine Angaben allerdings nur aus vorhandenen Bibliographien bezogen und die genannten Texte wohl nicht zur Hand gehabt zu haben. Auch die nächste Arbeit zu Mazepa in der deutschen Literatur stammt aus der Emigration - hier scheint das Thema besonders aktuell gewesen zu sein -, von einem der großen Gelehrten aus 
dem Ukrainischen Wissenschaftlichen Institut in Berlin, Dmytro Dorošenko, der 1941 sein bekanntes Buch Die Ukraine und das Reich. Neun Jahrhunderte deutschukrainischer Beziehungen im Spiegel der deutschen Wissenschaft und Literatur veröffentlichte (Doroschenko 1941). Im Kapitel „Die Ukraine in der deutschen schönen Literatur" nehmen die deutschsprachigen Mazepa-Bearbeitungen eine besondere Stellung ein. Dorošenko ist auch der erste, der auf die Mazepa-Reminiszenzen in Rilkes Gedicht Sturm aus dem Buch der Bilder hingewiesen hat (Doroschenko 1994: 285). 1974 verfasste Hubert F. Babinski ein ganzes Buch unter dem Titel The Mazepa Legend In European Romanticism (Babinski 1974), die bislang wohl umfangreichste Untersuchung zum Mazepa-Stoff in verschiedenen Literaturen, die aber einen großen Mangel hat: sie kennt keine einzige deutsche Mazepa-Bearbeitung; die europäische Romantik muss, so wie sie der Verfasser darstellt, ohne deren deutsche Komponente auskommen. Eine umfassende Studie aus neuerer Zeit stammt von Dmytro Nalyvajko aus dem Jahr 2002, sie trägt den Titel Mazepa v jevropejs 'kij literaturi XX st.: istorija ta mif, und geht ausführlich auf die deutschen Bearbeitungen ein (Nalyvajko 2002). Neue Wege bei der Deutung des Mazepa-Stoffs beschreitet Thomas Grob in seinem Aufsatz Der innere Orient. Mazeppas Ritt durch die Steppe als Passage zum anderen Europas (2005), der in dieser Figur vor allem ein „Narrativ der Grenzüberscheitung“ (Grob 2005: 37) sieht; in bezug auf die deutschen Bearbeitungen steuert Grob eine intertextuelle Mazepa-Variante in einer Erzählung von Gottfried Keller bei.

Das Interesse an Mazepas Nachleben in der deutschen Literatur besteht also weiterhin, eine umfassende Einordnung der deutschsprachigen Bearbeitungen in den Gesamttext der Mazepa-Literatur steht nach wie vor aus. Allerdings gilt auch heute noch, was Dorošenko schon 1941 konstatieren musste (Doroschenko 1941: 279): nicht alle der bibliographisch nachgewiesenen Mazepa-Bearbeitungen - mehr als ein Dutzend - sind in europäischen Bibliotheken vorhanden, unsere Untersuchung beschränkt sich also auf die Texte, die tatsächlich eingesehen werden konnten. Dabei tauchten aber auch Bearbeitungen auf, die bislang von niemandem erwähnt wurden; es ist also durchaus möglich, dass noch weitere deutsche Varianten des MazepaStoffs zu entdecken sind.

Generell gilt für die deutschen Bearbeitungen, was sich auch an den musikalischen Fassungen zeigte: sie gehen entweder auf die „westliche“, Byron'sche Fassung (die wiederum den Kern ihrer Erzählung von Voltaire übernommen hat) oder aber auf die „östliche“, russische Variante zurück, die mit Puškins Poem eine sehr wirksame Gestaltung erfahren hat.

\section{Im SChATTEN Byrons}

Byrons Mazeppa (1819), die letzte Verserzählung in der Reihe seiner Oriental Tales, hat wohl den literarischen Ruhm des ukrainischen Hetmans in der Romantik begründet; dieses Poem fand Übersetzer und Nachahmer in so gut wie allen europäischen Literaturen, von der spanischen bis zur slowenischen, und natürlich auch in 
der deutschen. Unter den Übersetzern ins Deutsche findet sich ein bekannter Name, Ferdinand Freiligrath (1810-1876), der sich im Völkerfrühling von 1848 hervortat. Seine Übersetzung ist ein Jugendwerk, das zu Lebzeiten des Dichters nie gedruckt wurde und erst posthum erschien. Was daran auffällt, ist zunächst der private Charakter: diese Übersetzung entstand zu einem Zeitpunkt, da es bereits zwei gedruckte deutsche Übersetzungen von Byrons Poem gab (die ersten beiden Übersetzungen ins Deutsche stammen aus den Jahren 1820 und 1821 - Rössing 1997: 64), es ging also nicht mehr darum, diesen Text dem deutschen Publikum zugänglich zu machen, sondern diese Übersetzung stellt vielmehr eine persönliche Auseinandersetzung des Dichters mit Byron und noch viel mehr mit Mazepa dar. Dabei scheint Byron für Freiligrath weniger wichtig gewesen zu sein als sein Held, denn obwohl der deutsche Dichter geradezu extensiv aus dem Englischen übersetzte, hat er nichts von Byron übersetzt - außer Mazeppa (Freiligrath 1909: 5 ff.). Es muss also eine Faszination gewesen sein, die von dieser Gestalt ausging und zur Auseinandersetzung mit ihr in Form einer Übersetzung führte. Beachtung verdient auch die Situation, in der die Übersetzung entstanden ist - sie hat manches mit Byrons Vorlage gemeinsam: bei einer Jagd zu Pferd legt der Dichter Freiligrath eine Rast ein, zieht eine billige Byron-Ausgabe aus der Satteltasche und beginnt spontan zu übersetzen (Freiligrath 1883: IV). Bei Byron machen Karl XII. und Mazeppa auf ihrer Flucht nach der Niederlage bei Poltava Rast, und Mazeppa beginnt zu erzählen. Der Ritt zu Pferd, der im Zentrum von Byrons Mazeppa steht, verbindet die Situation, aus der diese Übersetzung entstanden ist, mit dem zentralen Motiv der Vorlage.

Die spätere Begeisterung des Dichters für die Revolution des Jahres 1848, auf die Nalyvajko (2002: 16) und Doncov (1918: 24) verweisen, hat zum Zeitpunkt der Entstehung dieser Übersetzung fast zwanzig Jahre zuvor keine Rolle gespielt. Auch für Freiligrath - so wie für Byron - ist Mazepa ein Pole („Ha! Sie ist mein! - ach nur verstohlen / War ich der Glücklichste der Polen!“ - Freiligrath 1883: 20), der erst durch sein Geschick zum Fürsten der Ukraine wird. Die nationale Frage spielt hier sicher keine Rolle, im Vordergrund steht der Aufstieg des gedemütigten Pagen zum Herrscher eines Landes (,So schickte jener Thor, der Leiden / Für mich ersann ... mich nackt und blutend ins Verderben / Gebunden - um mir einen Thron / Jenseits der Steppe zu erwerben.“ - Freiligrath 1883: 49).

Freiligraths Übersetzung würde man heute eher als Nachdichtung, denn als wortgetreue Übersetzung bezeichnen, die Freiheit im Umgang mit dem Original führt bisweilen zu neuen Akzenten, wie etwa in den letzten Zeilen des Poems, da auch Mazeppa sich zum Schlafen legt, nachdem der König lang schon eingeschlafen ist: „Der Hetman legt mit frohem Sinn / Sich in der Eiche Schatten hin“ (Freiligrath 1883: 50) für „The Hetman threw / His length beneath the oak-tree shade“ (Vs. 860861 - Byron 1986: 200). Der „frohe Sinn“ fehlt im Original und würde auch nicht zu der von Byron gezeichneten Gestalt des Verlierers passen - Freiligrath verstärkt hier die optimistischen Töne, die mit der bevorstehenden Überquerung des Dnipro auch bei Byron gegeben sind. Seine Übersetzung bleibt aber dem Original in metrischer 
Hinsicht treu: es sind dieselben vierhebigen Jamben wie bei Byron, und was den Reim angeht, beschränkt sich Freiligrath auf Paarreime, während Byron auch alternierende Reime kennt. Ohne auf den Vergleich von Original und Übersetzung ausführlicher einzugehen, ist doch zu sagen, dass Freiligraths Nachdichtung eine sehr gelungene Übersetzung ist - es sind nur wenige Stellen, die man als misslungen bezeichnen würde, und es ist gut möglich, dass der Dichter diese Übersetzung erst dann veröffentlichen wollte, wenn er diese Schwächen verbessert haben sollte (Freiligrath 1883: V).

Ein völlig entgegengesetztes Modell einer Mazepa-Bearbeitung nach Byron stellt die Erzählung Mazeppa oder: Der schauerliche Ritt des schönen Edelknaben durch die fürchterliche Wildniß. Nach Lord Byron dem Volke erzählt von ... dar, deren Verfasser sich hinter dem Pseudonym „G. \& B. Ovm“ verbirgt. Dieser nicht datierte, in Linz (Oberösterreich) erschienene Text ist in der Sekundärliteratur völlig unbekannt, er gehört zu jenen Arbeiten, die im Zug der Recherchen zu dieser Untersuchung gefunden wurden. Von Byrons Poem hat diese Prosafassung nur die Story entlehnt, die man eigentlich schon seit Voltaires L'Histoire de Charles XII, roi de Suède (1731) kennt: die Romanze des Pagen Mazepa am Warschauer Hof mit einer polnischen Adeligen, die zur grausamen Bestrafung durch deren betrogenen Ehegatten führt: Mazepa, auf ein wildes Pferd gebunden, überlebt diesen Ritt und wird, halbtot, von ukrainischen Kosaken gefunden und gerettet.

So wird denn auch diese „Nacherzählung“ im Sinn einer „Schauergeschichte“ für die Bedürfnisse eines anspruchslosen Massenlesers zurechtgemacht: der Rahmen wird weggelassen, die komplexe Erzählstruktur Byrons vereinfacht. Der eigentliche Ritt Mazeppas - wenn auch im Titel noch genannt -, der bei Byron den größten Teil der Binnenerzählung ausmacht, wird zurückgedrängt, im Vordergrund steht eine breit ausgebaute Liebes- und Betrugsgeschichte (6 von 10 Kapiteln). Dafür wird die Geschichte über das von Byron gewählte Ende hinaus weiter erzählt, großteils aus der eigenen Phantasie. Im ersten Teil finden sich nur mehr einige wenige, mehr oder minder zufällige Reminiszenzen an Byron, etwa der Name Therese, während der ihres Mannes, Graf Lewinsky, erfunden ist. Das Dreiecksverhältnis Graf-ThereseMazeppa wird um die Person des Stallmeisters ergänzt, der, selbst in Therese verliebt, ihre Beziehung zu Mazeppa an den Grafen verrät. Auch die Bestrafung Thereses - ihr Mann lässt sie in Gegenwart Mazeppas grausam töten - ist frei erfunden. Mit der Bestrafung Mazeppas und seinem ungewollten Ritt auf Leben und Tod nähert sich der Verfasser dieser Schrift wieder der Byron'schen Vorlage. Das schlanke Kosakenmädchen, das auch bei Byron den Helden liebevoll gesund pflegt, ist bei unserem Verfasser die Tochter des Kosakenfürsten Sultanow (Ovm o. J.: 52), dessen Nachfolger Mazeppa quasi durch die Heirat mit dieser Tochter wird.

Interessant ist der dritte Teil, das 10. und letzte Kapitel dieser Erzählung „Mazeppas letzte Heldenthaten und sein Tod““. Zunächst nimmt Mazeppa Rache am Grafen Lewinsky - mit seinen Kosaken kommt er zurück nach Polen und lässt dessen Schloss verbrennen. Dann aber zieht er, schon „als achtzigjähriger Greis“ (Ovm 
o. J.: 55) mit Karl XII. gegen Peter I., um gegen diesen bei Poltava zu verlieren. Während die Schlacht von 1709 nur mit einem Satz erwähnt wird, wird Mazeppas Tod ausführlich geschildert - ganz anders, als wir das aus der Geschichte kennen: er ermöglicht es dem Schwedenkönig, den Dnipro zu überschreiten (für den Verfasser ist das bereits der Grenzfluss zum Osmanischen Reich!), indem er, heldenhaft kämpfend, die russischen Verfolger aufhält ${ }^{2}$. Als er schließlich selbst samt seinem Ross in den Fluss springt, um sich zu retten, wird er von russischen Kugeln getroffen und versinkt in den Fluten. Es ist ein seltsames Gemisch von „Dichtung und Wahrheit“, das uns in diesem letzten Kapitel der Erzählung begegnet: die Schlacht von Poltava samt dem genauen Datum, dem 8. Juli 1709 (Ovm o. J.: 55), die dramatischen Szenen, als der König mit dem kleineren Teil des schwedischen Heeres bei Perevoločnja den Dnipro überquert, das alles entspricht dem historischen Befund, i. U. zu Mazeppas Rache in Polen und zu seinem Tod in den Fluten. Die Frage nach den Quellen, denen der Verfasser diese Informationen entnommen hat, ist nur schwer zu beantworten, auch weil wir das Jahr der Entstehung seines Textes nicht kennen. Ein kleiner Hinweis auf andere Mazepa-Bearbeitungen findet sich am Ende des Textes: „Ja der Name Mazeppa lebt noch heute und wird in ewiger Zeit fortleben in den Liedern der Kosaken - und Polen. In Deutschland und Ungarn aber erzählt man in Prosa den Jünglingen und Jungfrauen von dem schönen Edelknaben und seinem entsetzlichen Ritt durch die Wildniß“ (Ovm o. J.: 58).

Ohne Zweifel auch der Byron'schen Vorlage verpflichtet, ohne diese aber im Titel oder sonst irgendwo zu nennen, ist Gustav Nieritz' (1795-1876) Erzählung Mazeppa, 1842 im Berliner Volks-Kalender erschienen. Auf diese Erzählung stützte sich offenbar Bert Brecht, als er in seine Sammlung Hauspostille (1921) auch eine kurze Ballade Mazeppa aufnahm (Brecht 1988: 319). Auch Nieritz lässt den historischen Rahmen von Byrons Poem weg - von Poltava und Karl XII. findet sich keine Spur - und transferiert die gebundene Sprache in Prosa. Er lässt seine Handlung um die Mitte des 17. Jahrhunderts im Warschauer Königsschloss beginnen, wo sich der Page Mazeppa in die schöne Helene verliebt (und sie in ihn), von einem Kammerherrn, dem Grafen Rutowsky, aber daran gehindert wird. Der Streit zwischen dem Pagen und dem Kammerherrn direkt vor den königlichen Gemächern, bei dem der König („Sr. Majestät, König Kasimir“ - Nieritz 1842: 123) selbst vermitteln muss, erinnert an den ältesten Bericht über Mazepa in den Pamiętniki des Jan Chrysostom Pasek aus dem 17. Jhd. - dass Nieritz diese seit 1836 veröffentlichten Memoiren kannte, ist sehr zu bezweifeln.

Die Heirat des älteren, reichen Grafen mit der jungen Helene kommt nur zustande, weil dieser ihr vorgemacht hat, Mazeppa sei nicht mehr am Leben, und damit

${ }^{2}$ Der heldenhafte Widerstand der Kosaken gegen eine gewaltige russiche Übermacht bei der Überquerung des Dnipro findet sich in einer ukrainischen belletristischen Mazepa-Bearbeitung des 20. Jhds. im 5. Bd. von Bohdan Lepkyjs Mazepa-Roman. Z pid Poltavy do Bendery (New York 1955). 
seinem Rivalen zuvorgekommen ist (eine solche Entwicklung der Intrige wird zwanzig Jahre später von Karl Kösting in seinem Trauerspiel Die Nebenbuhler aufgegriffen). Hier wird Byrons und damit auch Voltaires Vorlage deutlich modifiziert, um Mazeppa zu rehabilitieren: nicht er hat die Frau eines anderen verführt, sondern er wird von einem anderen um seine Liebe betrogen; Mazeppa ist nicht der Betrüger, sondern der Betrogene. Erst nach der vom Grafen erschlichenen Heirat kommt es zum Verhältnis zwischen Mazeppa und der jetzigen Gräfin (von Nieritz ebenso knapp geschildert wie bei Byron, im Unterschied zur obigen Erzählung), zu dessen Aufdeckung und zur Bestrafung Mazeppas in der bekannten Weise. Dabei wird der unfreiwillige Ritt Mazeppas ziemlich ausführlich und in deutlicher Anlehnung an Byron gestaltet, seine Rettung durch die Kosaken aber nur knapp erwähnt (auch das Kosakenmädchen, das den Todkranken gesund pflegt, fehlt in dieser Erzählung). Die Erzählung schließt mit einer ausführlichen Schilderung der Rache Mazeppas am Grafen, dessen Schloss er von seinen Kosaken verbrennen lässt - der Graf selbst kommt in den Flammen um, seine unglückliche Frau war schon zuvor an Gram gestorben. Mit dem Satz „Mazeppa erlebte das späteste Greisenalter; doch vermählt hat er sich nimmer" (Nieritz 1842: 136) endet diese Mazepa-Variante, von der Niederlage bei Poltava oder auch dem Tod des Protagonisten ist keine Rede. Diese Erzählung ist nicht nur weitgehend a-historisch, sondern auch a-politisch gehalten: Mazeppas ukrainische Abstammung kommt ebenso wenig zur Sprache wie sein politisches Programm, das er als Führer der Kosaken zu verwirklichen hätte.

Auf Byrons Vorlage geht auch Karl Köstings (1842-1907) Drama Die Nebenbuhler. Trauerspiel in vier Aufzügen (1886) zurück, das im Unterschied zur Erzählung von Gustav Nieritz sehr wohl eine politische Mission Mazeppas kennt, ansonsten aber stark an dessen Gestaltung der Handlung erinnert. Kösting hatte bereits 1861 eine Tragödie in fünf Akten, Mazeppa oder das Geheimnis der Steppe verfasst, die aber nie gedruckt wurde (Grodeke 2005: 461); 25 Jahre später überarbeitete er dieses Jugendwerk und ließ es drucken - auch ihn scheint Mazepa ein Leben lang beschäftigt zu haben. Im Zentrum der Handlung steht einmal mehr eine Liebesintrige, diesmal aber zwischen zwei Gleichaltrigen: Mazeppa und Graf Laremba bemühen sich beide um Guselda, ein armes Mädchen von edler Abstammung, das im Schloss des Grafen erzogen wird. Weil Guselda aber den armen Mazeppa und nicht den reichen Grafen bevorzugt, beschließt dieser, den Nebenbuhler aus dem Weg räumen zu lassen - auf die von Byron bekannte Weise. Auf dem Rücken des wilden Pferdes gerät Mazepa bis in die Sič und wird dort aufgrund seiner Tapferkeit zum Anführer der Kosaken gewählt. Mit diesen Kosaken kehrt Mazeppa nach Polen zurück, um das geliebte Mädchen zu holen und am Grafen Rache zu nehmen. Weil er aber nur die Rache am Nebenbuhler im Sinn hat, kann er den Selbstmord Guseldas, die inzwischen Larembas Frau werden musste, nicht verhindern. An der Bahre der Toten versöhnen sich die Nebenbuhler, Laremba nimmt Gift, Mazeppa, durch diese Erfahrung gereift und geläutert, ist nun für seine eigentliche Mission bereit. 
Diese eigentliche Mission Mazeppas wird im Stück nicht mehr gezeigt, das allerdings mit seinen für die Tragödie ungewöhnlichen vier Akten eine Art offenes Ende bietet (die erste Fassung hatte noch 5 Akte!). Aus Mazeppas Monologen im Kosakenlager (2. Akt) lässt sich diese Mission aber gut ablesen: Mazeppa, durch göttliche Fügung zum Zaporoger Kosaken geworden (von Geburt ist auch Köstings Held ein Pole), soll zum Anführer einer christlichen Streitmacht werden, welche den Islam aus Europa verdrängt. Hier wird - so absurd es auch klingt - ein Vergleich mit dem sog. Eroberer Sibiriens, Ermak Timofeev, bemüht, um die christliche Mission Mazeppas herauszustellen: so wie Ermak das Kreuz in den hohen Norden gebracht hätte, so soll Mazeppa es auf der Hagia Sophia aufrichten: „Trug er glorreich / Das Kreuz bis zu des Nordpols Silbereis - / Wer wollt' euch hindern, wenn er wiederkäme, / Das Christenzeichen auf der goldnen Kuppel / Der Hagia Sophia aufzupflanzen? (Kösting 1886: 27). Hier wird ein Stück zaristisch-panslawistischer russischer Ideologie dem ukrainischen Hetman in den Mund gelegt, was sich wohl aus der Entstehungszeit des Dramas erklären lässt: Köstings gedruckte Fassung erschien kurz nach dem russisch-türkischen Krieg von 1883, in dem die Eroberung Konstantinopels/Stambuls ein erklärtes Kriegsziel zumindest der russischen Propaganda war.

Köstings Drama löst Mazepa fast ganz aus dem historischen Kontext - von Jan Kazimierz, Karl XII., Peter I. oder Poltava ist nie die Rede -, es bringt aber eine moralische Rehabilitierung, die schon bei Nieritz zu spüren war, und eine Idealisierung Mazeppas. Als Werkzeug der göttlichen Vorsehung kann Mazeppa nicht aus eigenem Willen handeln, er ist vielmehr ein Berufener, vergleichbar mit einer alttestamentarischen Führergestalt, die den Willen Gottes tun muss. Seine Errettung von dem wilden Ritt ist ein Hinweis darauf: „Der Weg allein / Auf dem mich Gott zu meinem Amt geführt, / Macht mich besingenswerth, Gott offenbart / Nur an Berufnen sich durch solche Wunder!“ (Kösting 1886: 36). Für diese Berufung aber bedarf es - wie auch bei vielen biblischen Vorbildern - einer moralischen Läuterung. Mazeppa, geleitet vom blinden Hass auf seinen Nebenbuhler, kann nicht der künftige Führer seines Volkes sein. Erst durch den Tod der Geliebten, an dem Mazeppa selbst mitschuldig ist, und über die Versöhnung mit seinem Feind erfolgt die moralische Wiedergeburt Mazeppas: „... ein Läut'rugnsfeuer / Brennt nun in mir! [zu den Kriegern] Wollt ihr aus dem Metall / Das hier sich läutert, euch den Hetman formen, / Auf den ihr hofft?“ (Kösting 1886: 65). Die letzte Replik Mazeppas, bevor der Vorhang fällt - „In dieser Stunde ward er [der Hetman - A. W.] euch geboren!“ erinnert nicht nur an die letzten Zeilen von Victor Hugos Poem („Ce cadavre vivant, les tribus de l'Ukraine / Le feront prince un jour" Vs. 92 f. - Hugo 1954: 147), sie bietet eine messianistische Deutung Mazeppas, die allerdings erst dann wirksam werden kann, wenn das Drama der persönlichen Leidenschaften zu Ende ist. Das durch die vier Akte des Dramas gegebene offene Ende weckt beim Zuschauer Interesse für die zukünftige Mission dieses Hetmans, gleich, ob er um die Niederlage bei Poltava weiß oder nicht. 


\section{IM SCHATTEN PUŠKINS}

Eine zweite Gruppe von deutschen Mazepa-Bearbeitungen steht zumindest in typologischer Hinsicht unter dem Einfluss von A.S. Puškins Poltava, seinem Poem aus dem Jahr 1829, das ja ursprünglich auch Mazepa heißen sollte. Auch wenn Puškin als Vorlage nicht explizit genannt wird, so geht diese Abhängigkeit aus der Story, die auf der Liebesbeziehung des alten Mazepa zu Matrona (bei Puškin: Marija), der Tochter seines Freundes Kočubej, sowie des doppelten Verrats (Kočubej denunziert Mazepa bei Peter, Mazepa wiederum hintergeht den Zaren) beruht, hervor. Dabei ist anzumerken, dass die deutschen Bearbeiter nicht unbedingt auch Puškins Drama gekannt haben müssen, sie konnten diese Intrige auch aus einer der Hauptquellen, auf die sich Puškin stützte, übernommen haben: eine deutsche (Teil-)Übersetzung von Dmitrij Bantyš-Kamenskijs Istorija maloj Rossii stand ab 1835 zur Verfügung (Dorošenko 1994: 279). Ebenso wichtig wie diese Abhängigkeit ist aber etwas Anderes: in ihrer Beurteilung der Person Mazepas weichen die deutschen Autoren diametral von der Verurteilung, die Mazepa bei Puškin als „Verräter“ erfährt, ab. Mehr als hundert Jahre zuvor war in der deutschen Publizistik das Gegenteil zu beobachten: die positiven Darstellungen Mazepas in führenden deutschsprachigen Zeitschriften wurden nach 1708 mit einem Schlag negativ, die meisten deutschen Medien übernahmen den russischen Standpunkt (Krupnyc'kyj 1932: 310 ff.). Dass der russische offizielle Standpunkt bei westeuropäischen Schriftstellern und Intellektuellen nach 1848 nicht sehr geschätzt wird, verwundert nicht - vielleicht ist auch das ein Grund für das Interesse an Personen, die Opfer der russischen Großmachtpolitik geworden waren.

Am Beginn der deutschen Mazepa-Rezeption nach Puškin steht - so wie im Fall Byrons - eine Übersetzung, die schon 1831, also zwei Jahre nach dem Original, erschienen ist. Allerdings ist dieser Text, der im Dezember 1831 in der Leipziger Zeitschrift Der Komet. Ein Unterhaltungsblatt für die gebildete Leserwelt unter dem Titel Pultava. Eine Erzählung abgedruckt wurde, mit keinem Wort als Übersetzung bezeichnet und auch auf Puškin als den Autor gibt es keinerlei Hinweis (der Text scheint deswegen auch in keiner Übersetzungsbibliographie auf - Reissner 1970: 368 ff.). Dazu kommt, dass der Übersetzer, der sich hinter dem Pseudonym S.H.T. verbirgt, Puškins Poem ganz einfach in Prosa transferiert hat - deshalb „Erzählung“ im Untertitel. Man braucht aber nur die ersten Zeilen zu lesen, um zu sehen, dass es sich um eine fast wortwörtliche Übertragung handelt, die nur bisweilen etwas kürzt. Ein Beispiel vom Beginn dieser Erzählung: „Kotschubei ist reich und berühmt. Das Auge kann die Ausdehnung seiner Wiesen nicht ermessen, auf denen seine zahlreichen Herden weiden“ (S.H.T. 1831: 1617); im Original: „Богат и славен Кочубей. / Его луга необозримы; / Там табуны его коней / пасутся вольны, нехранимы.“ (Vs. 1-4 - Puškin 1948: 19). Der Verfasser dieses Plagiats - so müsste man es nach heutiger Auffassung wohl nennen - setzt aber keine eigenen Akzente in der Bewertung Mazepas, die seine Übertragung vom Original unterscheiden würden. 1831, sieben Jahre vor seinem Tod, war A.S. Puškin dem deutschen Leser noch weitge- 
hend unbekannt - vielleicht ist das der Grund, warum er als Verfasser von Pultava nicht genannt wird. Ungeachtet dessen scheint S.H.T. der erste zu sein, der die „östliche" Variante der Mazepa-Erzählung in den deutschen Umlauf gebracht hat.

Andreas May (1817-1899) ist als Dramatiker heute so gut wie vergessen, sein Drama Der König der Steppe, 1849 in München uraufgeführt, gilt bei den wenigen Interpreten als ein schwaches Werk (Lewickyj 1904: 614; Doncov 1917: 288 f.; Dorošenko 1994: 279). Dennoch lohnt es sich, dieses Stück vor dem Hintergrund der Puškin'schen Folie zu betrachten, auch wenn Puškins Poem aufgrund der Abgesetztheit des 3. Liedes zu einer offenen, Mays „Drama in fünf Aufzügen“ aber zu einer geschlossenen Form neigt. In der Konstellation der handelnden Figuren gibt es Parallelen, die nicht zu übersehen sind, wie die Liebe Mazeppas zu Natalie, der Tochter seines Freundes Kotschubej, die zum Konflikt zwischen Vater und Tochter einerseits, aber auch zwischen Mazeppa und seinem alten Freund und Kampfgenossen führen muss. Eine zweite Konstellation erinnert an Puškin: in Mays Drama wirbt ein junger Adliger, Fürst Dimitri Galizin, um Kotschubejs Tochter, findet aber bei ihr kein Gehör; das erinnert an jenen namenlosen Kosaken in Poltava, der, ebenso hoffnungslos in Marija verliebt, die Denunziation ihres Vaters nach Moskau bringt und bei der Schilderung der Schlacht von Poltava noch einmal auftaucht, um - ebenso vergeblich - Marija zu rächen und Mazepa zu töten. Auch könnte man in der Dienerin und Vertrauten Natalies, Eudoxia, ein Pendant für die Mutter Marijas bei Puškin sehen.

Was die Handlung von Mays Drama betrifft, so entsprechen die Akte 1, 2, 3 und 4 durchaus dem Verlauf der Handlung der ersten beiden Lieder von Poltava: Mazeppa hat sich in Kotschubejs Tochter verliebt und sie entführt, sie ist von ihm begeistert, weil sie in ihm den künftigen König der Ukraine sieht; Kotschubej berichtet von Mazeppas geplantem Übertritt auf die schwedische Seite nach Moskau, dort aber schenkt man ihm keinen Glauben, Peter lässt ihn gefangen an Mazeppa übergeben. Auch der folgende Konflikt der jungen Frau, die zwischen Vater und Geliebtem wählen muss, ist bei May genau so dramatisch gestaltet wie bei Puškin. In Mays Drama ist die Heldin selbst sich der Notwendigkeit dieser Wahl bewusst (,Sei's Gatte - Vater - Einen muß ich wählen!“ - May 1849: 84), in Poltava stellt Mazepa selbst seine Gattin vor diese Wahl: („Скажи: отец или супруг / Тебе дороже?“ Песнь II, Vs. 96 - Puškin 1948: 37). Damit ist aber auch eine andere Weiterentwicklung der Intrige bei beiden Autoren gegeben: während Puškins Marija nicht mehr wählen kann - Mazepa ist ihr zuvorgekommen und hat ihren Vater hinrichten lassen -, zögert Mays Natalie nicht, dem Gatten einen Giftbecher zu verabreichen, um den Vater zu retten. Auch wenn dieses Gift nur ein Schlaftrunk war, der ihr die Chance gibt samt ihrem Vater aus Mazeppas Schloss zu fliehen, so hat sie sich dennoch eindeutig gegen den Gatten und damit auch gegen dessen politisches Programm entschieden, von dem sie anfänglich so begeistert war. Die weibliche Hauptfigur wird im Vergleich zu Puškins Vorlage deutlich umgestaltet - sie endet auch nicht im Wahnsinn. 
Auch die militärisch-politische Niederlage Mazeppas in Poltava wird von May ausgeblendet (so wie in Čajkovskis Oper). Mazeppa wird am Ende dieses Dramas standrechtlich erschossen - von russischen Truppen, die seine Residenz Baturin eingenommen haben. Auch wenn May hier (und anderswo) eklatant gegen die historische Wirklichkeit verstößt (Lewickyj 1904: 614), ist nicht anzunehmen, dass er diese nicht gekannt hätte, denn manche der Repliken seiner handelnden Personen verraten eine gute Kenntnis der Details etwa von der Einnahme und Zerstörung Baturins („Und Baturin ist in meiner Russen Hand. Geleitet von bestochenen Kosaken / Drang ich mit meinem Regimente durch / Geheime Pforten ein“ - May 1849: 95; „Es ist des Czaren Wille, daß kein Stein / hier fürder auf dem andern haften bleibe“ - May 1849: 96). Warum also der Verstoß gegen die historische Wahrheit im Großen, wenn man ihr im Kleinen treu bleibt? Die Tragödie braucht den Tod des Protagonisten, um das Scheitern von dessen Programm zu zeigen; der wirkliche Tod Mazeppas im Exil wäre hier zu wenig aussagekräftig.

Im Mazeppas Programm aber liegt der größte Unterschied zwischen Mays Drama und Puškins Poem (das stellenweise auch dramatisierte Passagen aufweist), die Deutung der Figur des ukrainischen Hetmans, die der deutsche Autor gibt, legt eine Polemik mit Puškin nahe (so wie dieser mit Ryleev und Byron polemisiert hatte), der bei aller künstlerischen Qualität seines Poems in Poltava doch eine offizielle, russisch-staatliche Auffassung vertritt und diese gerade mit seinem Poem sehr publikumswirksam gemacht hat. Mays Drama hingegen ist ein deutlich ,,promazepianisches Werk" (Nalyvajko 2002: 15), sein Mazeppa kommt der ukrainischen Sicht auf Mazepa sehr nahe. Mazeppas erster großer Monolog im 1. Akt lässt daran keinen Zweifel:

Es sei denn. Kotschubej,

Du kennst das lose Band, durch welches die

Ukraine mit dem Czarenreich verknüpft ist.

Freiwillig nur, des eig'nen Schutzes halber

Hat mein Kosakenvolk sich dereinst unter

Rußlands Botmäßigkeit begeben. Es

Versprach dem Czaren Waffendienst; der Czar

Schwor ihm dagegen, seine Freiheiten

Und Privilegien aufrecht zu erhalten.

Dies Schutzverhältnis wurde bald zur Bürde.

Der Moskowite hat sich nicht gescheut, Kopfsteuern und Accisen einzuführen,

Die uns'ren Rechten stracks zuwiderlaufen.

Längst murrt' das Volk und trug nur im Gefühl'

Vergeb'nen Widerstandes Rußlands Joch.

D i e Zeit soll aus sein, Bruder Kotschubej.

Ein freier Staat soll die Ukraine werden, 
Ein freies Reich soll hier entsteh'n, selbständig

Und unabhängig! Wie ein Riese soll es

Sich zwischen Osten und dem Westen lagern,

Ein Bollwerk gegen Rußland für Europa!

Freund, ein Kosakenreich, ein Königreich

Der Steppe! - Und erräthst du, wen dies Reich

Bald seinen ersten König nennen wird?

(May 1849: 11 f.)

Nicht nur Kotschubej, auch der Leser bzw. Zuschauer errät, dass dieser König Mazeppa heißen soll - prompt lässt sich dieser auch im 3. Akt von den Seinen huldigen, einmal mehr in krassem Widerspruch zu den historischen Fakten. Die Argumentation Mazeppas in diesem Monolog verweist auf eine sehr gute Kenntnis der historischen Umstände bzw. der Begründung für den Übertritt auf die schwedische Seite. In diesem Zusammenhang muss auch der Vorwurf des Verrats, von Puškin in Poltava zementiert, entkräftet werden: dort, wo von Seiten des Schutzherrn bestehende Verträge nicht eingehalten und ständig verletzt werden, hat auch der Vasall das Recht, sich von seinen Pflichten zu befreien. „Hat nur der Herrschende das Recht, Verträge zu brechen?“ (May 1849: 14) fragt Mazeppa pointiert und rechtfertigt damit sein Vorhaben vor seinen Anhängern. Sein Übertritt auf die schwedische Seite ist also nicht Verrat, sondern zulässige Folge eines nicht eingehaltenen Bündnisvertrags, eine Argumentation, die für Puškin völlig undenkbar wäre. Mays Drama scheint gerade auf dem Hintergrund dieser Unterschiede doch von größerem Interesse zu sein, als üblicherweise angenommen; es zeugt von einer deutlichen Sympathie des Verfassers für die Ukraine, die auch dort erhalten bleibt, wo er sich an eine russische Vorlage anlehnt.

Fast zwanzig Jahre nach Mays König der Steppe erschien Rudolf v. Gottschalls (1823-1909) Mazeppa. Geschichtliches Trauerspiel in fünf Aufzügen (1865), das auf diversen Bühnen schon seit 1860 in leicht veränderter Form gespielt wurde. Es hat in der Sekundärliteratur am meisten Beachtung gefunden, von der fundamentalen Kritik durch Alfred Jensen ${ }^{3}$ über das Lob von Seiten Doncovs ${ }^{4}$ bis zu einer moderaten Anerkennung von Seiten Dorošenkos (Dorošenko 1994: 279 ff.) und Nalyvajkos (Nalyvajko 2002: 16). Auch die Übersetzung dieses deutschen Mazepa-Dramas ins Ukrainische durch Jurij Osyp Fed'kovyč (zwischen 1872 und 1874) trug zur besonderen Beachtung von Gottschalls Mazeppa bei. Für Ivan Franko, den Herausgeber von Fed'kovyč' dramatischen Werken, war diese Übersetzung Anlass zu einer ver-

3 „Dieses schreckliche Zufallsdrama macht auf die heutigen Leser beinahe einen groteskkomischen Eindruck mit seinen lächerlichen Verkehrtheiten“ (Jensen 1909: 302).

4 „Der deutsche Dichter hat die historische Rolle Mazeppas vollkommen richtig eingeschätzt und begriffen ..." (Donzow 1918: 26). 
nichtenden Kritik von Gottschalls Original ${ }^{5}$. Gottschall selbst, der schon 1850 in seinem Poem Gonta das erste Mal auf ukrainische Themen zurückgegriffen hatte und zu Lebzeiten ein erfolgreicher Bühnenautor war, ist heute so gut wie vergessen, von seinen vielen Stücken findet sich kein einziges mehr im Repertoire der deutschen Theater. Sein Mazeppa ist auch deshalb von Interesse, weil dieses Stück quasi eine Synthese der Byron'schen und der Puškin'schen Variante darstellt, was von den Interpreten bisher überhaupt nicht gesehen wurde - diese haben lediglich auf eine Beeinflussung des Autors durch Schillers Wallenstein hingewiesen (Doncov 1917: 287; Nalyvajko 2002: 16; Cieśła 1973: 199).

Zuvor aber noch zur historischen Zuverlässigkeit dieses Stücks, das im Untertitel Geschichtliches Trauerspiel heißt. Auch für Gottschalls Dramaturgie gilt, was von May gesagt wurde: die Manipulation historischer Fakten durch den Autor steht im Dienst einer anders verstandenen historischen Wahrheit. So finden wir auf den ersten Blick eklatante Verstöße gegen diese - Mazeppa hat eine Tochter namens Lodoiska, Matrena ist nicht die Tochter Kočubejs (der bei Gottschall überhaupt nicht vorkommt), sondern die Tochter Iskras, Mazeppa wird - ähnlich wie bei May - von seiner Geliebten vergiftet, dieses Mal aber wirklich, u. a. m). Bei einem genaueren Hinsehen finden wir eine gute Vertrautheit des Autors mit den historischen Umständen: Gottschall weiß von Kontakten Mazeppas zu den Don-Kosaken (deren Oberst Bulawin kommt im Stück vor), er weiß um die genauen Manöver Karls XII., er weiß, dass der Zar in der Schlacht von Poltava nur den Rang eines Obersten bekleidete und vieles andere mehr. Wenn Gottschall also gegen die historische Wahrheit verstößt, dann nicht aus Unkenntnis, sondern mit einer bestimmten Absicht.

Was die Intrige dieses Trauerspiels betrifft, so bleibt Gottschall bei dem Modell, das Puškin vorgegeben hat: Mazeppa hat Matrena, die Tochter Iskras, zu seiner Geliebten gemacht, Iskra will sich rächen und denunziert Mazeppa beim Zaren; dieser glaubt dem Denunzianten nicht, sondern liefert ihn an Mazeppa aus, welcher ihn hinrichten lässt (auch hier bleibt Gottschall seiner Vorlage treu, es gibt keine Rettung des Vaters durch die Tochter wie im Drama von May). Erst mit der Rache der Tochter, welche den ehemaligen Geliebten vergiftet, um den Vater zu rächen, weicht Gottschall von Puškin ab. Die Schlacht bei Poltava (bei Gottschall: „Pultawa“) bleibt bis auf eine kurze Szene ausgeblendet, auf das tragische Ende von Mazeppa und Matrena folgt ein glückliches: Lodoiska, Mazeppas Tochter, und Soldansky, (erfundener) polnischer Verbindungsmann zwischen Mazeppa und Karl XII., dürfen heiraten.

Auf motivisch-thematischer Ebene findet sich aber auch der Rückgriff auf Byrons Mazeppa. Gottschalls Titelheld selbst erzählt seiner Tochter von der Liebesaf-

5 „Безмірно довге балаканє, дешеве, професорське філозофованіє і повна імпотенція там, де треба б виявити якусь драматичну акцію ...“”(Franko 1902: X f.). 
färe mit der polnischen Dame und seiner grausamen Bestrafung, vom mörderischen, unfreiwilligen Ritt auf dem wilden Pferd (Gottschall 1865: 62 ff.). Der Name des betrogenen polnischen Edelmanns ist laut Gottschall Sapieha (einmal mehr ein eklatanter Verstoß gegen die historische Wahrheit), und weil Soldansky gesteht, ein Sohn Sapiehas aus zweiter Ehe zu sein, verbietet Mazeppa seiner Tochter jede Verbindung mit diesem Polen. Erst muss Soldansky in der Schlacht von Poltava Mazeppa das Leben retten, damit dieser seinen Sinn ändert. So wird die Aufnahme der Byron'schen Variante auch sujetär wirksam.

Vielleicht noch interessanter ist die thematische Deutung des Byron'schen Topos. In der Höhle der Wahrsagerin Harpyna, einer ehemaligen Geliebten Mazeppas, hängt ein Bild, das den an den Pferderücken gefesselten Mazeppa zeigt (man denke an die zahlreichen Darstellungen von Vernet, Boulanger, Delacroix u. a.). Dieses Bild wird von der Wahrsagerin im Dialog mit Lodoiska aber nicht historisch, sondern symbolisch gedeutet: „,... sieh’ das große Bild - den Jüngling / Ans Roß gebunden! Dieses ist dein Vater - / Und nicht dein Vater blos, es ist der Mensch, / Den ein unbändig Wollen mit sich fortreißt!“‘ (Gottschall 1865: 135).

Damit sind wir bei einem zentralen Punkt der Mazeppa-Interpretation Gottschalls angelangt, die dieser auch im Nachwort noch einmal bekräftigt: „Es bedarf für den aufmerksamen Leser wol nicht der besonderen Erwähnung, daß der aufs Roß gebundene Mazeppa nicht blos die tathsächliche Vignette, sondern das ethische Symbol der Dichtung ist, das Symbol der wilden und blinden Leidenschaft, die ins Verderben stürzt" (Gottschall 1865: 191 f.). Während die „blinde Leidenschaft“ eher in den Bereich der romantischen Vorstellungen gehört (und sehr gut zu den Byron'schen Helden passt), geht Dmytro Doncov mit seiner Deutung des Gottschall'schen Helden in eine andere Richtung: das absolute Müssen, welches Mazeppas Handeln bestimmt („Denn in der Tiefe uns'rer Seele wohnt ein dunkles Müssen“ - Gottschall 1865: 30), sein Streben nach der Krone der Ukraine um jeden Preis („nur das Ziel, das höher wächst im Preis durch solche Opfer“ - Gottschall 1865: 153) seien nichts Anderes als der von Nietzsche beschriebene „Wille zur Macht“ (Doncov 1917: 286). Erst über diesen „Willen zur Macht“ kommt das politische Programm Mazeppas ins Spiel, das dieser mehrmals in seinen Reden erläutert.

Mazeppa, durch ein absolutes Streben nach Macht gekennzeichnet, erinnert aber auch an den Helden von Puškins Poem. Auch dieser Mazepa ist vom Ehrgeiz getrieben, wichtiger als alle anderen politischen und privaten Motive ist seine Eitelkeit, die nur mit der Königskrone befriedigt werden kann. Die Krone ist auch für Gottschalls Mazeppa das Ziel seines Wollens (vgl. Gottschall 1865: 112), die Motive dafür sind jedoch anders als bei Puškin, sie lassen Mazeppa als einen Vertreter der ukrainischen Unabhängigkeit erscheinen, auch wenn zunächst - so wie bei Puškin ein persönliches Motiv im Vordergrund steht: die Rache für eine Beleidigung durch den Zaren (,Am Bart gezupft - mißhandelt wie ein Knecht, / Der Hetman der Kosacken - Rache, Rache!“" Gottschall 1865: 49). 
Wichtiger aber sind gesamtukrainische Anliegen, welche Gottschalls Mazeppa bei seinem Handeln leiten: die Unabhängigkeit des Volkes, das bislang stets im Dienst Polens oder Russlands stand (,Wenn's nicht um dieser Völker Freiheit wäre / die stets für fremde Herr'n ihr Blut verspritzen, / Für Warschau bald und bald für Moskau stöhnen!“ - Gottschall 1865: 52) und die Errichtung eines eigenen Staates. Diese Ideen werden in gleicher Weise auch von Matrena vertreten, bevor sie sich von Mazeppa abwendet: „Das wird ein Riesenreich, das weit hinein / Sich in den Osten dehnt, ein Reich der Steppen, / Das zu den fernen Hochgebirgen reicht!“ (Gottschall 1865: 109). Aber diese gewaltige Dimension des neu zu schaffenden Staates hängt an einem besonderen Menschen, an einem besonderen „Willen zur Macht“: „Nicht nach den Grenzen sucht ein kühner Geist, / In's Freie, Unermess'ne stürmt er hin!“ (Gottschall 1865: 109).

Es wäre also zu einfach Gottschalls Mazeppa aus dem Freiheitspathos von 1848 zu deuten, auch wenn der Autor ein begeisterter Anhänger des Völkerfrühlings war und eine Reihe von Texten in dessen Sinn geschrieben hat. Zu Recht weist Michał Cieśła darauf hin, dass Gottschall zum Zeitpunkt, da er seinen Mazeppa schrieb, bereits ganz andere ideologische Positionen vertrat (Cieśła 1973: 195 f.) - nicht umsonst wurde er 1877 vom deutschen Kaiser in den Adelsstand erhoben. Das große Werk der Befreiung eines Volkes aus der Unterdrückung und der Schaffung eines eigenen Staates kann nur von einem Menschen mit den Qualitäten eines Prometheus (Doncov 1918: 24), oder, um es mit Nietzsche zu sagen, von einem „Übermenschen" geleistet werden. Gottschalls Drama bietet am meisten Material für eine Interpretation Mazeppas im Sinn des „Willens zur Macht“, Dmytro Doncov hat diese Interpretation auch auf dem Hintergrund seines eigenen ideologischen Programms entwickelt.

Doncov baut seine Mazepa-Interpretation aber nicht nur auf die Philosophie Nietzsches, er bemüht auch einen berühmten Autor der Renaissance, Niccolò Machiavelli. 1934 erscheint in der Buchreihe der von Doncov herausgegebenen Zeitschrift Vistnyk eine Übersetzung von Machiavellis Il Principe, zu der Doncov wiederum ein ausführliches Vorwort verfasst hat, in dem er die Bedeutung von Machiavellis Thesen für die politische Situation der Ukraine in dieser Zeit klar machen will („Ніколи наука Макіявеля не була така актуальна, як теперь“- Makijavel’ 1934: 5). Die Fürsten-, Führer- und Herrscherfigur, die Machiavelli entwirft, zeichnet sich einmal mehr durch ein unbedingtes Wollen, den Willen zur Macht, aus: „Єдина пружина всього, що $\epsilon$ і стає - творча людська воля““- Makijavel' 1934: 6). Und ein zweites von Machiavelli postuliertes Kriterium trifft auch auf Mazepa zu - die Kunst, im Kampf mit unterschiedlichen Gegnern unterschiedliche Strategien anzuwenden, bzw. sich je nach Bedarf zu verstellen, einmal Löwe und einmal Fuchs zu sein, um es mit Machiavellis Worten zu sagen. Wille zur Macht, Verstellungskunst und schließlich die Freiheit in der Wahl der Mittel, welche den Zweck heiligen - das sind Züge, die Dmytro Doncov zum ersten Mal an Mazepa entdeckt hat, die aber in jüngster Zeit von ukrainischen Autoren vermehrt betont werden. 
Im Lauf der letzten Jahre ist eine Reihe von Veröffentlichungen erschienen, die von einem neuen Interesse an Mazepa, einem der tragischen Verlierer von Poltava, zeugen. Im Zusammenhang damit steht die Frage nach der Bedeutung Mazepas für die Ukraine heute - mit seiner, wenn auch erst in der letzten Phase seiner Regierungszeit vollzogenen Entscheidung für ein politisches Bündnis mit dem Westen könnte man in ihm ein Gegengewicht zu Chmel'nyc'kyj sehen, der 1654 ja eine in ihren Folgen weitreichende Anbindung an den Nachbarn im Osten begründet hatte. Vor allem publizistische und belletristische Arbeiten über Mazepa zielen heute darauf, das Bewusstsein einer breiteren Leserschicht zu prägen.

Valerij Ševčuk (geb. 1939), bekannt als Verfasser zeitgenössischer, psychologischer Prosa, hat sich seit fast 20 Jahren in einer Reihe von Arbeiten mit Mazepa beschäftigt, er ist auch der Verfasser des vorläufig letzten Dramas über Mazepa, Brama smertel'noji tini (1995). In seinem Buch Prosvičenyj volodar. Ivan Mazepa jak budivnyčyj Kozac'koji deržavy i jak literaturnyj heroj (2006) finden sich Thesen, die Doncov anhand von Gottschalls Drama entwickelt hatte. Schon im Titel könnte man eine Anspielung auf Macchiavelli sehen - „volodar“ ist der Begriff, mit dem ital. „principe“ bereits 1934 ins Ukrainische übersetzt wurde. Mazepa wird in diesem Buch als überzeugter Anhänger der Thesen Machiavellis dargestellt, wobei die mit diesem Begriff verbundenen negativen Assoziationen umgedeutet werden. Ein ganzes Kapitel (,Ivan Mazepa - polityk-makijavelist“ - Ševčuk 2006: 171-194) widmet der Verfasser diesem Thema, um zu beweisen, dass Mazepa in seinem politischen Handeln immer wieder den Ratschlägen des Italieners gefolgt sei, ob es um die Härte gegen innere Feinde geht, um die Demagogie im Umgang mit den Massen, um die Billigung eines jeden Mittels, das dem Ziel der Erhaltung eines starken Staates förderlich ist.

Mazepa als überzeugter Anhänger Machiavellis - das wirft auch auf den Standardvorwurf des „Verrats“, typisch vor allem für die russische Sicht Mazepas, ein neues Licht. Auch diesbezüglich schlägt Ševčuk eine aus dem deutschen MazepaDrama des 19. Jhds. bekannte Argumentationslinie ein - nicht Mazepa ist der Verräter, sondern Peter bzw. die russischen Regenten vor ihm, die systematisch die der Ukraine in den Verträgen von Perejaslavl' zugestandenen Rechte vorenthalten und verletzt hätten - so wie das schon Andreas May 1849 sieht. Die Suche nach einem anderen Protektor für die ukrainischen Sonderrechte und der damit verbundene Übergang auf die Seite Karls XII. seien also eine Sache gewesen, die Mazepa zwar im Sinn einer machiavellistischen Politik bis zuletzt geheim gehalten hätte, die aber im Sinn der alte Rechte und des staatlichen Selbstverständnisses der Ukraine völlig gerechtfertigt gewesen sei.

Es ist nicht uninteressant, diese engagierte ukrainische Darstellung Mazepas mit einer russischen zu vergleichen, der Biographie Mazepa von Tat'jana Tairova-Jakovleva, die als Publikation der Reihe Žizn' zamečatel'nych ljudej eindeutig populärwissenschaftlichen Charakter hat, auch wenn sie von einer Fachhistorikerin stammt. Schon in der Einführung stellt die Verfasserin ihr Anliegen vor: sie will 
dem Leser ein umfassendes Bild dieser Persönlichkeit geben, das es ihm ermöglicht, über die Stereotype ,izmennik“ bzw. „,geroj“ hinweg- und zu einer differenzierteren Beurteilung zu kommen (Tairova-Jakovleva 2007: 5). Auch wenn sich die beiden Bücher von ihrer Konzeption und vom Umfang her beträchtlich unterscheiden, sticht doch eine Gemeinsamkeit ins Auge: auch dieses Buch enthält ein Kapitel ,učenik Makiavelli“" (Tairova-Jakovleva 2007: 124-144), und die Prägung Mazepas durch die Lektüre von Il principe steht auch für Tairova-Jakovleva außer Zweifel, wenngleich sie den „Machiavellismus“ Mazepas doch anders fasst als Ševčuk: es sei Mazepa um die Durchsetzung einer Realpolitik, deren höchstes Ziel die Konsolidierung des Hetmanstaates war, gegangen. Die Zugeständnisse, die man dem russischen Oberherrn machen musste, passen voll und ganz in diese Politik.

Ganz ähnlich sieht die russische Historikerin die Problematik des Verrats. Mazepa hätte in der Situation der zunehmenden Konflikte mit Russland häufig auf vertraglich festgehaltene Rechte gepocht, die russische Seite hätte sich in der Zeit des Nordischen Krieges immer über diese Bedingungen hinweggesetzt, „Соответственно, нарушение положений договора одной стороны освобожлала другую от своих обязательств» (Tairova-Jakovleva 2007: 189). In einem solchen europäischen Rechtsverständnis liegt auch schon eine erste Antwort auf die Frage nach dem Verrat Mazepas.

Damit kommt Tairova-Jakovleva zu einer ganz eindeutigen Bewertung dessen, was in der russischen Geschichte seit fast 300 Jahren als Verrat gilt: nicht die Logik des Verräters, sondern die des Politikers hätte Mazepa zu diesem Schritt veranlasst, um das Schlimmste für sein Land - die Verwüstung durch schwedische wie auch russische Truppen - zu verhindern, und sie vergleicht diesen Schritt mit dem Frieden von Altranstädt, den August II. mit Karl XII. einige Jahre vorher geschlossen hatte, ohne übrigens seinen Bundesgenossen Peter davon in Kenntnis zu setzen: „Мазепа, по сути, просто повторил путь Августа, поставив интересы своего края и своего народа выше союзнического долга“ (Tairova-Jakovleva 2007: 219). Es ist eine späte Rehabilitierung Mazepas, die in diesem Buch vorgenommen wird, und die vieles mit der ukrainischen Sicht gemeinsam hat - ein seltener Fall bei der Bewertung einer gemeinsamen Geschichte aus zwei unterschiedlichen nationalen Traditionen.

In Bezug auf die Rehabilitierung Mazepas vom Vorwurf des Verrats decken sich zeitgenössische, populärwissenschaftliche und historische Arbeiten aber auch mit dem Bild, das die deutsche Literatur des 19. Jhds. vom Hetmann entworfen hat und es ist erstaunlich, wie sehr sich die Argumente gleichen, die Mazepa vom Vorwurf des Verrats befreien. Als ein Mann von europäischer Bildung, der die Ukraine außenpolitisch an Westeuropa binden und sie dazu aus russischer Abhängigkeit befreien wollte, gewinnt Hetman Ivan Mazepa heute neue Bedeutung für die Ukraine. 


\section{L i t e r a t u r}

Babinski 1974:

Brecht 1988:

Byron 1986:

Cieśła 1973:

Doncov 1917:

Donzow 1918:

gart), 20. Jahrgang, Nr. 13, 23-27

Doroschenko 1941/1994: Dmytro Doroschenko, Die Ukraine und das Reich. Neun Jahrhunderte deutsch-ukrainischer Beziehungen im Spiegel der deutschen Wissenschaft und Literatur, Leipzig; Neuauflage (1994) unter dem Titel: Die Ukraine und Deutschland. Neun Jahrhunderte Deutsch-Ukrainischer Beziehungen, München

Franko 1902:

Freiligrath 1883:

Freiligrath 1909:

Geyer 2009:

Gottschall 1865:

Grob 2005:

Grodeke 2005:

Hugo 1954:

Jensen 1909:

Juščenko 2007:

Kösting 1886:

Lewickyj 1904:

Krupnyc'kyj 1932:

Mac'kiv 1988:

Makijavel' 1934:

Hubert F. Babinski, The Mazeppa Legend in European Romanticism, New York

Kommentar zu Hauspostille, in: Bertolt Brecht, Werke. Große kommentierte Berliner und Frankfurter Ausgabe. Hg. v. Werner Hecht, Jan Knopf, Werner Mittenzwei, Klaus-Detlef Müller, Bd. 11: Gedichte I. Sammlungen 1918-1938, Berlin u. Weimar - Frankfurt a. M.

Lord Byron, The Complete Poetical Works. Ed. By Jerome J. McGann, Volume IV, Oxford

Michał Cieśła, Rudolf Gottschall i jego dramat historyczny Mazepa, Slavia Orientalis. Rocznik XXII, Nr. 2, 189-200

Дмитро Донцов, Гетьман Мазепа в європейській лїтературі, Шляхи (Львів), річник IV (1917), 283-291

Dmytro Donzow, Mazeppa in der Weltliteratur, Der Stürmer (StuttПисаня Осипа Юрія Федьковича. Перше повне і критичне виданє. Драматичні переклади Осипа Юрія Федьковича. 3 автографів уперве видав Др. Іван Франко, У Львові

Nachwort von I(da) F(reiligrath) in: Nachgelassenes von Ferdinand Feiligrath, Stuttgart, III-VIII

Freiligraths Werke in sechs Teilen. Hg. v. Julius Schwering. Fünfter Teil: Übersetzungen II (Übersetzungen aus dem Englischen), Berlin Leipzig - Wien - Stuttgart

Dietrich Geyer, Es begann in Poltava, Die Zeit Nr. 23 vom 28.05.2009 Mazeppa. Geschichtliches Trauerspiel in fünf Aufzügen von Rudolf Gottschall, Leipzig

Thomas Grob, Der innere Orient. Mazeppas Ritt durch die Steppe als Passage zum Anderen Europas, Wiener Slawistischer Almanach 56, $33-86$

Deutsches Schriftsteller-Lexikon 1830-1880 (Grodekes Grundriss zur Geschichte der deutschen Dichtung. Fortführung), I-K, Berlin

Victor Hugo, Les Orientales. Édition critique avec une introduction, des notices, des variantes et des notes par Élisabeth Barineau. Tome II, Paris

Alfred Jensen, Mazepa in der modernen europäischen Dichtung, Ukrainische Rundschau (Wien), Nr. 7, 299-305

Указ Президента України № 955/2007 про відзначення 300-річчя подій, пов'язанних 3 воєнно-політичнм виступом гетмана України Івана Мазепи та укладенням українсько-шведського союзу Karl Kösting, Die Nebenbuhler. Trauerspiel in vier Aufzügen, Darmstadt

Wassyl Lewickyj, Der Hetman Mazepa in der deutschen Literatur, Ruthenische Revue (Wien), Jahrgang II, Nr. 21, 596-644

Борис Крупницький, Гетьман Мазепа в освітленню німецької літератури його часу, в: Записки чина св. Василия Великого / Analecta ordinis S. Basilii Magni (Жовква). T. IV. Вип. 1-2, 292-316 Теодор Мацьків, Гетьман Іван Мазепа в західньоєвропейських джерелах 1687-1709, Мюнхен

Никола Макіявель, Володар (= Книгозбірна Вістника ч. 2), Львів 
May 1849:

Der König der Steppe. Drama in fünf Aufzügen von A. May, Mün-

Muchyn 1932: chen

Nalyvajko 2002:

Михайло Мухин, Гетьман Іван Мазепа в світовій літературі та мистецтві, в: Книголюб (Прага), кн. I - II, 3-10

Nieritz 1842:

Дмитро Наливайко, Мазепа в європейській літературі XIX ст.: історія та міф, Слово і час № 8, 39-48; № 9, 3-17

Ovm o. J.: Mazeppa. Eine Erzählung von Gustav Nieritz, in: Volks-Kalender, Berlin, 120-136

G. \& B. Ovm, Mazeppa oder: Der schauerliche Ritt des schönen Edelknaben durch die fürchterliche Wildniß. Nach Lord Byron dem Volke erzählt von G. \& B. Ovm, Linz

Puškin 1948: $\quad$ А. С. Пушкин, Полное собрание сочинений. Издательство АН CCCP, т. V: Поэмы, Ленинград

Reissner 1970: $\quad$ Eberhard Reissner, Deutschland und die russische Literatur 18001848, Berlin

Rössing 1997: Wolfgang Rössing, Literaturen der Welt in deutscher Übersetzung. Eine chronologische Bibliographie, Stuttgart - Weimar

Ševčuk 2006: $\quad$ Валерий Шевчук, Просвічений володар. Іван Мазепа як будівничий козацької держави і як літературний герой, Київ

S.H.T. 1831: $\quad$ Pultava, in: Der Komet. Ein Unterhaltungsblatt für die gebildete Leserwelt. Hg. v. C. Herloßsohn (Leipzig), Zweiter Jg., 1617-1662

Sičyns'kyj 1942: $\quad$ Володимир Січинський, Чужинці про Україну, Прага

Tairova-Jakovleva 2007: Татьяна Таирова-Яковлева, Мазепа. Жизнь замечательных людей, Москва

A bstract: Ivan Mazepa in German-language literature. Works on the Ukrainian Hetman Ivan Mazepa in the English (G. Byron's Mazeppa), French (V. Hugo's Mazeppa) and Russian (A. Pushkin's Poltava) romantic literatures are well known; numerous variations of the Mazepa-plot in the German literature, however, are completely forgotten today. This study shows how German playwrights such as Karl Kösting, Andreas May and Rudolf v. Gottschall as well as novelists like Gustav Nieritz, G. \& B. Ovm and S.H.T. borrow from Byron's $M a-$ zeppa on the one hand and from Pushkin's Poltava on the other, usually without quoting the original. In the first case the story of Byron's poem is remarkably inflated; in the other, the main hero, hetman Mazepa, is exculpated from the accusation of betrayal, formulated in Pushkin's Poltava. A similar positive treatment of Mazepa's role, as found in the German works examined, can be seen in recent Ukrainian publications (for example in a book by Valeryj Shevchuk from 2006).

K e y w o r d s : Mazepa, German Literature, Byron, Pushkin

Alois Woldan

Institut für Slawistik der Universität Wien

Universitätscampus AAKH, Hof 3

Spitalgasse 2, 1090 Wien, Österreich

alois.woldan@univie.ac.at 